\title{
Optical lymph node detection system: A practical device to assist lymph node location in neck resection specimens
}

\author{
YUJIA WANG $^{1 *}$, YUMEI PU $^{1 *}$, YANHONG NI $^{2}$, ZHIYONG WANG ${ }^{1}$, \\ XIAOFENG HUANG ${ }^{3}$, GUOWEN SUN ${ }^{1}$ and QINGANG HU ${ }^{1}$ \\ Departments of ${ }^{1}$ Oral and Maxillofacial Surgery, ${ }^{2}$ Central Laboratory and ${ }^{3}$ Oral Pathology, Nanjing Stomatological Hospital, \\ Medical School of Nanjing University, Nanjing, Jiangsu 210008, P.R. China
}

Received August 24, 2017; Accepted January 24, 2018

DOI: $10.3892 / \mathrm{ol} .2018 .7973$

\begin{abstract}
The status of lymph node(LN) metastasis, including the number and location of positive LNs, is a significant prognostic factor for oral squamous cell carcinoma (OSCC). Therefore, knowing the number and location of positive LNs is essential for prognosis. However, surgeons often have difficulty locating LNs. In the present study a practical device to improve the location of LNs in neck resection specimens was intoduced: the optical lymph nodes detection system (OLNDS). With the assistance of the OLNDS LNs were easier to locate and a significantly higher number of LNs were identified $(\mathrm{P}=0.006)$. The false detection rate was also significantly reduced compared with the traditional method $(\mathrm{P}=0.0034)$. The OLNDS was observed to be a valuable adjunct to the traditional method and may be useful for studying the value of $\mathrm{LN}$ metastasis in OSCC prognosis.
\end{abstract}

\section{Introduction}

Approximately 640,000 patients with oral squamous cell carcinoma (OSCC) are diagnosed each year worldwide, with a rising incidence in many countries (1). Despite evolution in its management, the overall survival (OS) rate has not improved significantly during the past 20 years, with 5-year survival rates between 45 and 50\% (2). Pathological lymph node (LN) metastases $\left(\mathrm{pN}^{+}\right)$are recognized as an adverse prognostic factor in OSCC (3). LN-associated factors, such as the number of positive nodes, the presence of extracapsular spread (ECS),

Correspondence to: Dr Qingang Hu or Dr Guowen Sun, Department of Oral and Maxillofacial Surgery, Nanjing Stomatological Hospital, Medical School of Nanjing University, 30 Zhongyang Road, Nanjing, Jiangsu 210008, P.R. China

E-mail: qghu@nju.edu.cn

E-mail:238957@sina.com

${ }^{*}$ Contributed equally

Key words: oral squamous cell carcinoma, lymph node metastasis, prognosis the number of nodes with ECS, the presence of contralateral neck metastases ( $\mathrm{pN} 2 \mathrm{c})$, the presence of lower neck metastases (level IV/V), the number of dissected LNs and the LN density may influence survival rates (4-6). In this context, the number and location of all retrieved LNs from the neck dissection (ND) specimen is of critical importance to the diagnosis of the number, ratio, and location of positive LNs.

At present, to retrieve LNs from an ND sample, surgeons can only rely on their sense of touch or the color difference between superficial LNs and the surrounding tissue. However, such techniques can easily overlook small and/or deep LNs. Another problem is that once the specimen is removed from the patient, it is hard to determine the location of the LNs, especially when resected by less experienced surgeons.

Here we introduce a straightforward device, named optical lymph nodes detection system (OLNDS), to improve LNs location accuracy detection rate. Our experience had demonstrated that it could be useful in identifying and locating LNs, especially for less experienced surgeons.

\section{Materials and methods}

Patients. An ND specimen from each of 63 patients (Group 1) who had primary surgery for OSCC with ND (radical or selective) in the Department of Oral and Maxillofacial Surgery, Nanjing Stomatological Hospital, Medical School of Nanjing University between November 2014 and May 2015 were evaluated with the OLNDS in addition to the traditional method. LNs were isolated from the ND specimen by a qualified resident doctor or a qualified attending doctor. The data of another 70 patients (Group 2) were retrieved from our database. These patients went through primary surgery for OSCC with ND (radical or selective) in the same department between January 2011 and December 2012. Their ND specimens were subjected only to the traditional LN location method by the same attending doctor who participated in the study. Ethical approval had previously been obtained from the Nanjing Stomatological Hospital Research Ethics Committee. Written informed consents were obtained from participants before delivery.

Patient information is summarized in Table I. In brief, among patients in Group 1, 37 (57.8\%) were male and $26(42.2 \%)$ female. Patient ages ranged between 29 and 89 years 
(mean \pm SEM, 59.52 \pm 1.418$)$. In Group 2, 30 (42.6\%) were male and $40(57.4 \%)$ were female. Patient ages ranged between 35 and 81 years (mean \pm SEM, 59.06 \pm 0.349 ).

The OLNDS consists of two parts: the LN localization board and the LN detection light box.

The LN localization board. The LN localization board is a transparent acrylic board printed with black neck division guidance. The white area in Fig. 1A is transparent, while the black is printed as black to review neck division and block out extraneous light. It can be used together with a light blockage board (Fig. 1B) to suit different ND tissue requirements. The board can also be printed as in Fig. 1C, using a different color to block extraneous light. Magnets are inlayed into the board (as indicated by red spots in Fig. 1D) to stretch (make it thinner) and to fix the ND specimen. The LN localization board can be sterilized and be used during the operation, so that the chief surgeon can put the ND specimen onto the board directly to ensure the accuracy of its location.

The LN detection light box. A lamp is installed on the base of the LN detection light box and a piece of highly transparent glass is used to place the positioning plate. A switch in front of the box allows for the brightness of the lamp to be adjusted (Fig. 1E). During the operation, the chief surgeon placed the ND specimen directly onto the board with accurately located specimen. The ND specimen was then searched for LNs without the assistance of OLNDS. When no more LNs could be found with the traditional method, the specimen was searched for LNs under the guidance of OLNDS. The number of LNs from each region by different methods was recorded. The diameter of the smallest LN found by the two different methods was measured with vernier calipers.

Statistical analysis. The paired Student's t-test was used to compare the difference between the number and smallest diameter of the LNs found by different methods. The level of significance was set at $5 \%(\mathrm{P}<0.05)$. Statistics were analyzed with Prism 5.0.

\section{Results}

OLNDS helped to reveal LNs. When the light was off, only superficial LNs were seen (black arrow indicating superficial LN; Fig. 2A). When the light was turned on, even deeper LNs were clearly revealed as round dark spots (white arrow; Fig. 2B), while superficial LNs were clearer than when the light was off (black arrow; Fig. 2B). To distinguish LNs (white arrow; Fig. 2C) from thick tissue (black arrow; Fig. 2C), LNs appeared to be round and uniform, while the thick tissue had a rough outline and was not evenly dark inside. Moreover, the dark spot of thick tissue would disappear when squeezed or stretched, while the LNs would not.

As the light was strong, directly viewing it could obscure the tissue; therefore, unnecessary light was blocked, letting only necessary light pass the tissue. This was accomplished with a light-blockage board (Fig. 1B) or a light-blockage printed area (Fig. 1C). Both the light-blockage board and the light-blockage area can be shaped to fit different ND specimens. The OLNDS was found to be a powerful tool for
Table I. Clinical pathologic characteristics of patients with oral squamous cell carcinoma.

\begin{tabular}{lcc}
\hline & \multicolumn{2}{c}{ Status of the lymph nodes } \\
\cline { 2 - 3 } Clinical variable & Group 1 & Group 2 \\
\hline Total & 63 & 70 \\
Sex & & \\
Male & 37 & 40 \\
Female & 26 & 30 \\
Age (years) & & \\
$<60$ & 27 & 33 \\
$\geq 60$ & 36 & 37 \\
Type of neck dissection & & 56 \\
Radical & 45 & 14 \\
Selected & 18 & \\
Positive lymph nodes & & 34 \\
Yes & 19 & 45 \\
No & 44 & 24 \\
TNM stage & & 46 \\
Early stage & 36 & \\
Late stage & 27 & \\
Histopathology grading & & \\
I & & \\
II-III & & \\
\hline
\end{tabular}

locating and identifying LNs, especially for less experienced resident doctors.

As the LN localization board can be used during the operation and as the surgeon can place the ND specimen immediately after dissection, the location of the tissue can be highly accurate. In this context, even a resident doctor can locate the LNs accurately.

In addition to accurate location, in Group 1, OLNDS also helped identify more LNs than the traditional method $(\mathrm{P}=0.0006$; Fig. 3). The statistical significance was higher for the resident doctor $(\mathrm{P}=0.0042)$ than the attending doctor $(\mathrm{P}=0.0206)$. Again, compared with data retrieved (Group 2), OLNDS found more LNs (mean, 6.01) than with the traditional method only (mean, 2.29) in each region.

OLNDS could detect smaller LNs compared with the conventional method. For 21 patients in Group 1, the diameter of the smallest LNs found by different method (traditional or with OLNDS) were measured with Vernier calipers. With the traditional method, the average diameter of the smallest LNs detected was $3.18 \mathrm{~mm}$ (SEM, 0.28). With OLNDS, the average diameter of the smallest LNs detected was $2.02 \mathrm{~mm}$ (SEM, $0.21)$. The difference between these two groups was significant ( $\mathrm{P}=0.0001$; Fig. 4), indicating that smaller LNs, which could otherwise not be discovered by the traditional method, could be found with OLNDS.

$O L N D S$ reduced false detection rate. The LNs retrieved from the ND specimens of 64 patients in Group 1 were sent for pathological analysis to the Department of Oral Pathology, 

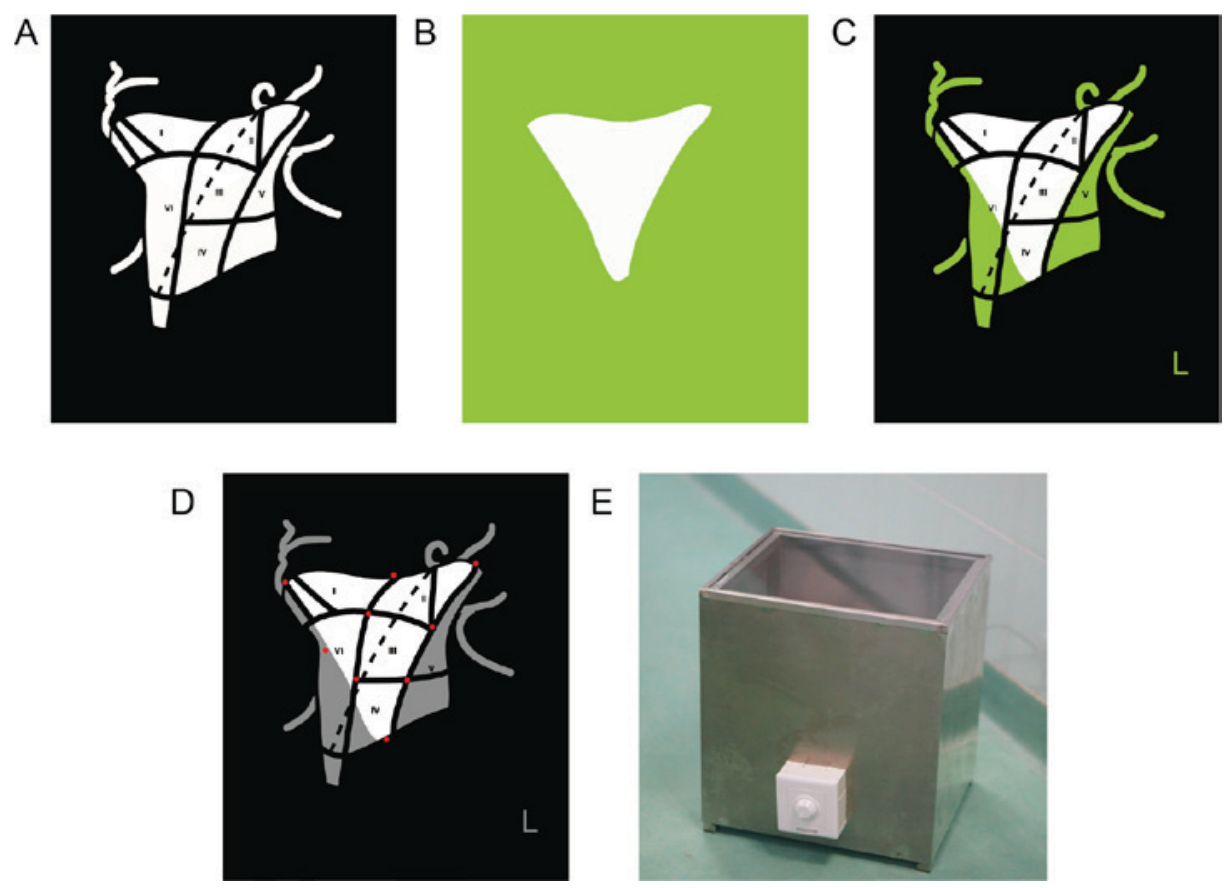

Figure 1. Optical lymph nodes detection system. (A) The neck division is printed on the acrylic board. To help block extra light, A can be used together with the (B) light blockage board or be printed as in (C). The transparent areas of (B) and (C) can be of various shapes to suit different kinds of neck dissection. Magnets are inlayed into the board (as indicated by red spots in D) to stretch (make it thinner for observation) and fix the neck dissection specimen. (E) The lymph node detection light box. The switch in the front can turn on/off the light and adjust the brightness. The top of the box is made of transparent glass to allow the light to go through.
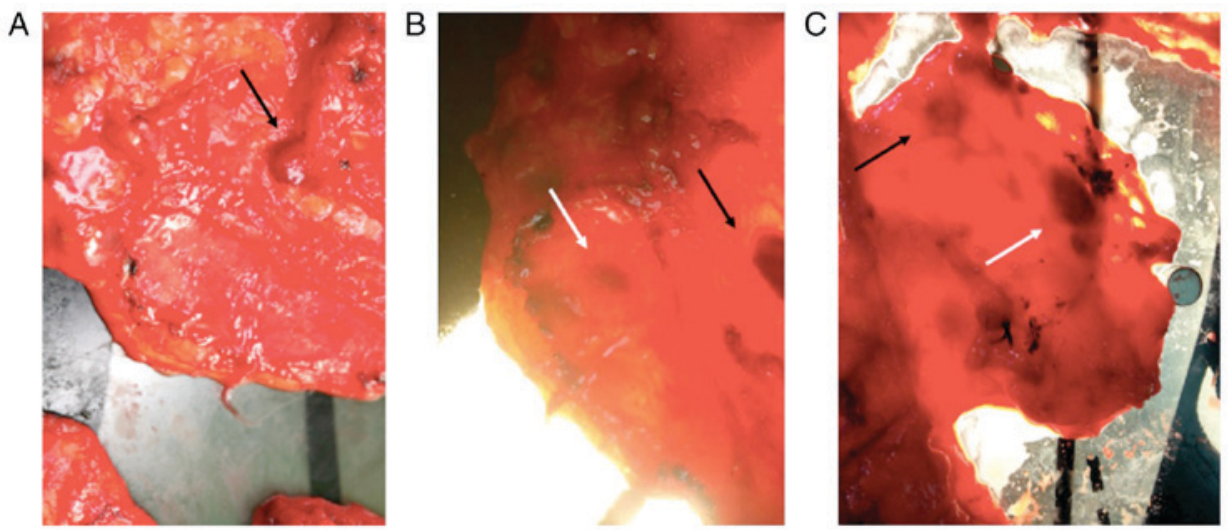

Figure 2. Optical lymph node detection system helps reveal LNs. (A) When the light is off, only superficial LNs can be seen (black arrow indicates superficial LN). (B) When the light is turned on, even deeper LNs revealed themselves clearly as round dark spots (white arrows), while superficial LNs were even clearer than when the light was off (black arrow). (C) To distinguish LNs (white arrow) from thick tissue (black arrow), LNs appear to be round and uniform, while the thick tissue had a rough outline and is not evenly dark inside. Moreover, dark spots of thick tissue disappear when squeezed or stretched, while LNs do not. LN, lymph node.

A

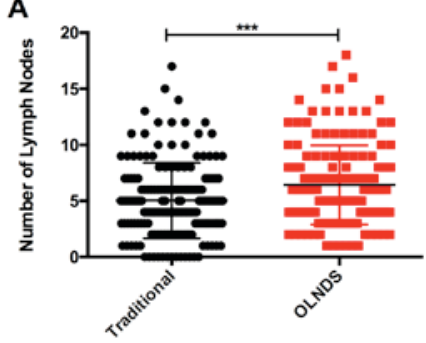

B

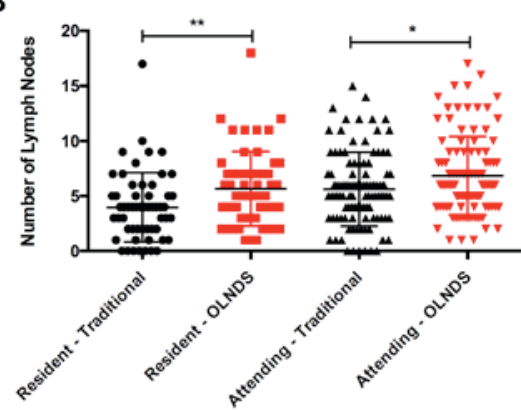

Figure 3. OLNDS helps find more LNs. (A) in Group 1, with the help of OLNDS, more lymph nodes were found ( $\mathrm{P}=0.0006)$. (B) OLNDS helped both resident and attending doctors find more LNs. Its value was more significant for resident doctors $(\mathrm{P}=0.0042)$ than for attending doctors $(\mathrm{P}=0.0206)$. OLNDS, optical lymph nodes detection system; LN, lymph node. ${ }^{*} \mathrm{P}<0.05,{ }^{* *} \mathrm{P}<0.005$ and ${ }^{* * *} \mathrm{P}<0.001$. 


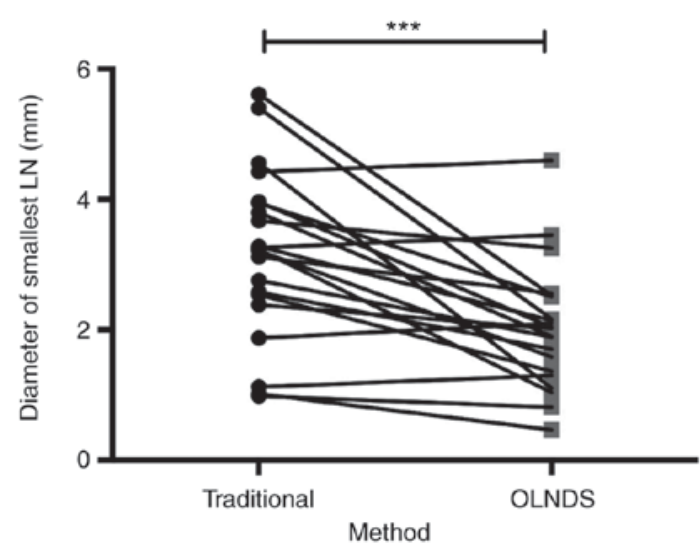

Figure 4. OLNDS can help find smaller lymph nodes than the traditional method (mean value was compared with paired Student's t-test, ${ }^{* * *} \mathrm{P}<0.001$ ). OLNDS, optical lymph nodes detection system; LN, lymph node.

Nanjing Stomatological Hospital, Medical School of Nanjing University. Among the 1,471 LNs found, 5 were not LNs (false detection ratio, 0.0034). Comparably, among 70 patients in Group 2, 943 LNs were isolated from ND specimens by the same attending doctor who participated in this experiment, among which 28 were not LNs (false detection ratio, 0.0296).

\section{Discussion}

The presence of LN metastasis has long been one of the most important prognostic factors for the survival of patients with OSCC (7). LN associated factors, including the number (or density) of LNs (8-10), the number (or ratio) of positive LNs and LNs with extracapsular metastasis (11-14), and the location of positive LNs or LNs with extracapsular metastasis $(6,13)$, were reported to be related to the prognosis of OSCC patients and could guide adjuvant therapy. According to the 8th edition of the TNM staging system, the number of positive LNs has become a crucial factor in determining the $\mathrm{N}$ category (15).

However, an accurate location and thorough separation of the LNs is essential. Unfortunately, once an ND specimen is removed from the patient, with the loss of anatomic references, it is difficult to divide the specimen accurately according to the neck division standard (16), especially for inexperienced doctors. Moreover, the traditional method of identifying LNs relied merely on differences of touch and color between the LNs and connective tissues. This method could lead to the incomplete separation of LNs and also result in false detection by mistaking adipose tissue for LNs.

As described earlier, the OLNDS was designed to locate LDS more accurately than the traditional method, especially for junior doctors. Further, magnets could be used to fix and stretch the specimen, thus making the tissue thinner for the light to come through. Light blockage board or LN localization board printed with light blockage area could block out unnecessary light. As the light was quite strong, by using different light blockage combination could ensure that light only came through the tissue, thus making it much easier for the surgeons to look directly at the tissue.

In our actual practice with OLNDS, the ND specimen was first submitted to the traditional LN separation procedure. When no more LNs could be found with the traditional procedure, the light was turned on and the same surgeon searched for LNs again with the help of OLNDS. In most of the patients, we found more LNs, which would otherwise have been missed, with OLNDS. This effect was more obvious for junior doctors, who often lack experience compared with senior doctors.

Further, with OLNDS, surgeons could find smaller LNs than with the traditional method. For small LNs hidden inside the specimen, it is natural that surgeons could not see or feel them. However, under the strong light of OLNDS, the small LNs revealed themselves as little dark spots.

Another problem with LN separation was false detection; in most cases, fat tissue was mistaken for LNs. With OLNDS, the false detection rate was reduced. Some fat tissue can be round like a LN, and some LNs can be light in color like fat tissue. With OLNDS, the difference can be visualized as LNs generally have a higher density. The major disadvantage of OLNDS is that it is more time-consuming than the traditional method alone. This is the challenge in applying the OLNDS.

The follow-up period of patients in group 1 was between 5 and 29 months (average 23 months). During this time, no recurrence was recorded. Seven patients died from OSCC, and 10 had metastasis. Among the 63 patients in group 1, the 2-year OS rate was $88.9 \%$, the 2-year recurrence-free survival rate was $100 \%$, and the 2-year metastasis-free survival (MFS) rate was $84.1 \%$. We reviewed 680 OSCC patients whose ND specimen was searched for LNs with the traditional method and found their 2-year OS rate was $90.9 \%$, the 2-year recurrence-free survival rate was $89.5 \%$, and the 2 -year MFS rate was $90.0 \%$. We attribute the seemingly different 2-year OS, RFS, and MFS rates to the comparatively small sample we included in this study. As the OLNDS was designed to complement the traditional method and was used only after no more LN could be found with the traditional method, it would only improve the separation of LNs. As we include more patients in the study and expand the follow-up period, we believe we can better design treatment for and improve the prognosis of OSCC patients.

In conclusion, even though OLNDS is never meant to be an independent LN separation method, it can be a powerful supplement to the traditional method and may significantly increase the location accuracy separation rate of LNs. As LN metastasis is an important prognosis-related factor not only of oral cancer but also melanoma, breast cancer, and other solid tumors (17-19), OLNDS can be used to deepen the study of LN metastasis of various cancers. Its value should be further verified by linking the accurate number and location of positive LNs with prognosis.

\section{Acknowledgements}

The optical lymph nodes detection system is protected by patent. Patent nos. ZL201520506264.4 (the lymph node localization board) and ZL201520506339.9 (the lymph node detection light box). This study was supported partly by the National Key Disciplines Constructional Project Funding, China, partly by Nanjing Municipal Key Medical Laboratory Constructional Project Funding (since 2012), and partly by the Center of Nanjing Clinical Medicine Tumor Project (since 2014). 


\section{References}

1. Parkin DM, Bray F, Ferlay J and Pisani P: Global cancer statistics, 2002. CA Cancer J Clin 55: 74-108, 2005.

2. Bagan JV and Scully C: Recent advances in oral oncology 2007: Epidemiology, aetiopathogenesis, diagnosis and prognostication. Oral Oncol 44: 103-108, 2008.

3. Woolgar JA, Triantafyllou A, Lewis JS Jr, Hunt J, Williams MD, Takes RP, Thompson LD, Slootweg PJ, Devaney KO and Ferlito A: Prognostic biological features in neck dissection specimens. Eur Arch Otorhinolaryngol 270: 1581-1592, 2013.

4. Liao CT, Hsueh C, Lee LY, Lin CY, Fan KH, Wang HM, Huang SF, Chen IH, Kang CJ, Ng SH, et al: Neck dissection field and lymph node density predict prognosis in patients with oral cavity cancer and pathological node metastases treated with adjuvant therapy. Oral Oncol 48: 329-336, 2012.

5. Shingaki S, Takada M, Sasai K, Bibi R, Kobayashi T, Nomura T and Saito C: Impact of lymph node metastasis on the pattern of failure and survival in oral carcinomas. Am J Surg 185: 278-284, 2003.

6. Woolgar JA: Detailed topography of cervical lymph-note metastases from oral squamous cell carcinoma. Int J Oral Maxillofac Surg 26: 3-9, 1997.

7. Yuasa-Nakagawa K, Shibuya H, Yoshimura R, Miura M, Watanabe H, Kishimoto S and Omura K: Cervical lymph node metastasis from early-stage squamous cell carcinoma of the oral tongue. Acta Otolaryngol 133: 544-551, 2013.

8. Amar A, Chedid HM, Rapoport A, Cernea CR, Dedivitis RA, Curioni OA and Brandão LG: Prognostic significance of the number of lymph nodes in elective neck dissection for tongue and mouth floor cancers. Braz J Otorhinolaryngol 78: 22-26, 2012.

9. Patel SG, Amit M, Yen TC, Liao CT, Chaturvedi P, Agarwal JP, Kowalski LP, Ebrahimi A, Clark JR, Cernea CR, et al: Lymph node density in oral cavity cancer: Results of the international consortium for outcomes research. Br J Cancer 109: 2087-2095, 2013.

10. Ampil FL, Caldito G and Ghali GE: Can the lymph node ratio predict outcome in head and neck cancer with single metastasis positive-node? Oral Oncol 50: e18-e20, 2014.

11. Liao CT, Wang HM, Chang JT, Ng SH, Hsueh C, Lee LY, Lin CH, Chen IH, Huang SF and Yen TC: Analysis of risk factors for distant metastases in squamous cell carcinoma of the oral cavity. Cancer 110: 1501-1508, 2007.
12. Shibuya Y, Hasegawa T, Akashi M, Shigeta T, Minamikawa T and Komori T: Oral squamous cell carcinoma with multiple neck metastases-cases with more than ten pathologically positive lymph nodes in the unilateral side. J Oral Maxillofac Surg 71: 793-797, 2013.

13. Ebrahimi A, Gil Z, Amit M, Yen TC, Liao CT, Chaturvedi P, Agarwal JP, Kowalski LP, Kohler HF, Kreppel M, et al: The prognosis of $\mathrm{N} 2 \mathrm{~b}$ and $\mathrm{N} 2 \mathrm{c}$ lymph node disease in oral squamous cell carcinoma is determined by the number of metastatic lymph nodes rather than laterality: Evidence to support a revision of the American Joint Committee on Cancer staging system. Cancer 120: 1968-1974, 2014.

14. Sayed SI, Sharma S, Rane P, Vaishampayan S, Talole $S$, Chaturvedi $P$, Chaukar D, Deshmukh A, Agarwal JP and D'Cruz AK: Can metastatic lymph node ratio (LNR) predict survival in oral cavity cancer patients? J Surg Oncol 108: 256-263, 2013.

15. Huang SH and O'Sullivan B: Overview of the 8th edition TNM classification for head and neck cancer. Curr Treat Options Oncol 18: 40, 2017.

16. Robbins KT, Clayman G, Levine PA, Medina J, Sessions R, Shaha A, Som P and Wolf GT; American Head and Neck Society; American Academy of Otolaryngology-Head and Neck Surgery: Neck dissection classification update: Revisions proposed by the American Head and Neck Society and the American Academy of Otolaryngology-Head and Neck Surgery. Arch Otolaryngol Head Neck Surg 128: 751-758, 2002.

17. Leong SP and Tseng WW: Micrometastatic cancer cells in lymph nodes, bone marrow, and blood: Clinical significance and biologic implications. CA Cancer J Clin 64: 195-206, 2014.

18. Balch CM, Soong SJ, Gershenwald JE, Thompson JF, Reintgen DS, Cascinelli N, Urist M, McMasters KM, Ross MI, Kirkwood JM, et al: Prognostic factors analysis of 17,600 melanoma patients: Validation of the american joint committee on cancer melanoma staging system. J Clin Oncol 19: 3622-3634, 2001.

19. Leong SP, Nakakura EK, Pollock R, Choti MA, Morton DL, Henner WD, Lal A, Pillai R, Clark OH and Cady B: Unique patterns of metastases in common and rare types of malignancy. J Surg Oncol 103: 607-614, 2011 